\title{
GROUP STRUCTURE AND PROPERTIES OF BLOCK IDEALS OF THE GROUP ALGEBRA
}

\author{
by WOLFGANG HAMERNIK
}

(Received 15 November, 1973)

1. Introduction. In this note relations between the structure of a finite group $G$ and ringtheoretical properties of the group algebra $F G$ over a field $F$ with characteristic $p>0$ are investigated. Denoting by $J(R)$ the Jacobson radical and by $Z(R)$ the centre of the ring $R$, our aim is to prove the following theorem generalizing results of Wallace [10] and Spiegel [9]:

THEOREM. Let $G$ be a finite group and let $F$ be an arbitrary field of characteristic $p>0$. Denoting by $B_{1}$ the principal block ideal of the group algebra $F G$ the following statements are equivalent:

(i) $J\left(B_{1}\right) \leqq Z\left(B_{1}\right)$,

(ii) $J\left(B_{1}\right)$ is commutative,

(iii) $G$ is p-nilpotent with abelian Sylow p-subgroups.

The equivalence of (i) and (iii) was shown in [9] and of course, Theorem 2 of [10] is an immediate consequence of the theorem above. Its proof involves a heredity property of the principal block discussed in $\$ 2$ as well as methods of Wallace [10] and finally a result of Morita [7] which is included with a short proof following from the other preliminaries. As a corollary Wallace's characterization of group algebras with commutative radicals is obtained and proved for arbitrary ground fields.

In the following all modules are finitely generated unitary right modules. To avoid trivial statements it is always assumed that the prime $p$ divides the order $|G|$ of the finite group $G$. $O_{p},(G)$ is the largest normal subgroup of $G$ with an order not divisible by $p$. The block ideals of the group algebra $F G$ are the uniquely determined as two-sided ideals indecomposable direct summands of $F G$. As identity elements of the block ideals, the block idempotents are exactly the primitive idempotents of $Z(F G)$. A block of $F G$ is a triple $B \leftrightarrow e \leftrightarrow \lambda$ consisting of the block ideal $B$, its identity element $e$, and the linear character $\lambda$ of $Z(F G)$ with $\lambda(e) \neq 0$. An indecomposable $F G$-module $M$ belongs to the block (ideal) $B$ if $M e \neq 0 . \quad B_{1} \leftrightarrow e_{1} \leftrightarrow \lambda_{1}$ is the principal block of $F G$ if $e_{1}$ does not annihilate the trivial simple $F G$-module. Concerning the further terminology we refer to Michler [5] and Curtis-Reiner[1].

I am indebted to Professor G. Michler for helpful discussions.

2. A heredity property of the principal block. Throughout this section $F$ is an arbitrary field of characteristic $p>0$ and $G$ is a finite group. First we collect some elementary facts. We recall that for $F G$-modules $M$ and $N$ the tensor product $M \otimes_{F} N$ is defined by

$$
(m \otimes n) g=m g \otimes n g \quad(g \in G, m \in M, n \in N) .
$$


LEMMA 2.1. Let $E$ be an FG-module of dimension one and let $S$ be a simple FG-module. Then $E \otimes_{F} S$ is a simple $F G$-module.

Proof. There is an $x \in E$ such that $E=x F$. If $M \neq 0$ is a submodule of $E \otimes_{F} S$, then every $m \in M$ can be written as $m=x \otimes y$ for a $y \in S$. Because of the action of $G$ onto $E \otimes_{F} S$, the set

$$
M_{1}=\{y \in S \mid x \otimes y \in M\} \neq 0
$$

is a submodule of $S$ and hence $M_{1}=S$. Since $E$ has dimension one it follows that

$$
M=\left\{x \otimes y \mid y \in M_{1}=S\right\}=E \otimes_{F} S,
$$

which proves the lemma.

Lemma 2.2. Let $X, Y, M$ be FG-modules with $X \leqq Y$. Then

$$
Y \otimes_{F} M / X \otimes_{F} M \underset{F G}{\cong} Y / X \otimes_{F} M .
$$

The proof is easily given choosing suitable $F$-bases in the modules.

Proposition 2.3. Let $U_{1}$ be the indecomposable projective $F G$-module associated with. the trivial representation (i.e. $U_{1} / U_{1} \cdot J(F G) \cong F$ ) and let $U$ be any indecomposable projective FG-module. If all composition factors of $U_{1}$ have dimension one, then the dimensions of all composition factors of $U$ are equal.

Proof. Set $U / U \cdot J(F G)=V$. It is well known that $U$ is a direct summand of the projective $F G$-module $U_{1} \otimes_{F} V$. Let

$$
U_{1}=U_{11}>U_{12}>U_{13}>\ldots>U_{1 n}=0
$$

be a composition series of $U_{1}$. By Lemmas 2.1 and 2.2

$$
U_{1} \otimes_{F} V=U_{11} \otimes_{F} V>U_{12} \otimes_{F} V>\ldots>U_{1 n} \otimes_{F} V=0
$$

is a composition series of $U_{1} \otimes_{F} V$ and all composition factors have the same dimension, namely $\operatorname{dim}_{F} V$. This proves the assertion.

An immediate consequence is

COROLlary 2.4. Let $F$ be an arbitrary field of characteristic $p>0$ and let $G$ be a finite group. The following statements are equivalent:

(i) All irreducible representations of $G$ over $F$ belonging to the same block have the same F-dimension.

(ii) All irreducible representations of $G$ over $F$ belonging to the principal block have $F$ dimension one. 
(iii) All composition factors of the indecomposable projective $F G$-module associated with the trivial representation have F-dimension one.

Proof. (iii) implies (i) by 2.3 bearing in mind the fact that the indecomposable projective modules in a block are linked.

DEFINITION. The group algebras of finite groups characterized by Corollary 2.4 are in the following called group rings with dimension property.

REMARK. If the underlying field $F$ is a splitting field for the group $G$ then $F G$ has the dimension property if and only if in the decomposition of any block ideal into indecomposable projective modules all isomorphism types appear with the same multiplicity. Morita [7] calls such rings quasi-primary decomposable.

In the following sections the subsequent observation of Spiegel[9] will be used.

Lemma 2.5 ([9]). Let $G$ be a p-solvable group and let $H=O_{p} \cdot(G)$. Then

$$
e_{1}=\frac{1}{|H|} \sum_{h \in H} h
$$

is the principal block idempotent of $F G$.

Proof. ([9]). $e_{1} F G \cong F G / H$ implies that $e_{1} F G$ is the principal block ideal because $F G / H$ is by Fong's Theorem $[2,3 \mathrm{~A}]$ indecomposable as a two-sided ideal.

3. Group rings with dimension property. In this section $K$ is always a splitting field of characteristic $p>0$ for all subgroups and factor groups of the finite group $G$ (e.g. $K$ can be chosen to be algebraically closed). By $2.4, K G$ has the dimension property if and only if the same is true for the principal block ideal of $K G$.

The next result, for which we give a short proof for the sake of completeness, is due to Morita [7]; for another proof see [4].

Proposition 3.1. Let $H=O_{p},(G)$ and let $P$ be a Sylow p-subgroup of $G$. The following statements are equivalent:

(i) $H P$ is normal in $G$ and $G / H P$ is abelian. $\dagger$

(ii) The principal block ideal $B_{1}$ of $K G$ has the dimension property.

(iii) $K G$ has the dimension property.

Proof. $(i) \Rightarrow(i i)$. Set $\bar{G}=G / H$ and $\bar{P}=H P / H \cong P$. By hypothesis, $\bar{G}$ is a group with normal Sylow $p$-subgroup $\bar{P}$ and abelian factor group. Hence $K \bar{G}$ has only irreducible representations of dimension one. In particular $K \bar{G}$ has the dimension property. By 2.5 , $B_{1} \cong K \bar{G}$ and (ii) is proved.

$\dagger$ For example, these properties are satisfied if $G$ is supersolvable. 
(ii) $\Leftrightarrow($ iii). This follows from 2.4 .

(iii) $\Rightarrow(i)$. (cf. Morita [7], p. 188) With the notation of the first part of this proof $B_{1} \cong$ $K \bar{G}$. Since all irreducible representations of $B_{1}$ have dimension one, the right regular representation $\widetilde{G}$ of $\bar{G}$ on $K \bar{G}$ consists of lower triangular matrices. It is easily verified that exactly those of these triangular matrices with only elements equal to one in the main diagonal form a Sylow $p$-subgroup $\widetilde{P}$ of $\widetilde{G} \cong \bar{G}$. Furthermore $\widetilde{P}$ is normal in $\widetilde{G}$ and $\widetilde{G} / \widetilde{P}$ is abelian. This proves (i).

The following lemma is an immediate consequence of the fact that every group ring over a field is a symmetric algebra.

Lemma 3.2. Let $B \leftrightarrow e \leftrightarrow \lambda$ be a block of the group algebra $F G$ over an arbitrary field $F$ with positive defect. Then $J(B)^{2}=0$ if and only if there exists an (up to isomorphisms) unique projective indecomposable B-module of composition length 2.

The next result is due to Wallace[10]. Its proof is included for the sake of completeness.

LEMMA $3.3([10])$. If the radical $J=J(B)$ of the block ideal $B$ of the group algebra $K G$ is commutative, then either $J(B)^{2}=0$ or every composition factor of an arbitrary indecomposable projective $B$-module has $K$-dimension one.

Proof. If $J(B)^{2} \neq 0$, let $V$ be an indecomposable projective $B$-module. Since $K G$ is a quasi-Frobenius ring, it is sufficient to show that the socle soc $V$ of $V$ has dimension one. By $3.2 V \cdot J^{2} \neq 0$. Thus every element of soc $V$ is a linear combination of elements $x \cdot y$ with $x, y \in J$. Let $g, h$ be arbitrary elements of $G$. Then

$$
(x y)(g h)=x[(y g) h]=[(x h) y] g=(y x)(h g)=(x y)(h g) .
$$

This implies, by Burnside's theorem $([1], 27.4)$, that

$$
\operatorname{End}_{K}(\operatorname{soc} V)=\operatorname{End}_{K G}(\operatorname{soc} V) \text {, }
$$

and hence, by Schur's lemma, that

$$
\operatorname{End}_{K}(\operatorname{soc} V) \cong K
$$

Therefore $\operatorname{dim}_{K}(\operatorname{soc} V)=1$, and the lemma is proved.

PROPOSITION 3.4. If the radical $J\left(B_{1}\right)$ of the principal block ideal $B_{1}$ of the group algebra $K G$ is commutative, then $K G$ has the dimension property.

Proof. Since the principal block has highest defect, then $J\left(B_{1}\right) \neq 0$. By 2.4 , it suffices to show that all composition factors of an indecomposable projective module $V$ belonging to the principal block have dimension one. Hence the assertion follows from 3.3 and 3.2. 
4. Proof of the theorem.

(A part of the step (ii) $\Rightarrow$ (iii) is a modification of an argument in [10, p. 2]).

(i) $\Rightarrow$ (ii) clear.

(ii) $\Rightarrow($ iii). Let $K$ be the algebraic closure of $F$. Then

$$
K \otimes_{F} B_{1}=B_{1}^{*} \otimes \ldots \otimes B_{s}^{*},
$$

with block ideals $B_{1}^{*}, \ldots, B_{s}^{*}$ of $K G$; we may assume that $B_{1}^{*}$ is the principal block ideal of $K G$. Since $F G / J(F G)$ is a separable algebra (cf. [5],12.11), we conclude that

$$
K \otimes_{F} J\left(B_{1}\right)=J\left(K \otimes_{F} B_{1}\right)
$$

by $[5,12.12]$. Hence $J\left(B_{1}^{*}\right)$ is commutative also. By $3.2, K G$ has the dimension property. Let $H=O_{p},(G)$ and let $P$ be a Sylow $p$-subgroup of $G$. 3.1 implies that $A=H P$ is normal in $G$ and that $G / H P$ is abelian. It remains to show that $H P=G$. Because $G$ is $p$-solvable, 2.5 yields that

$$
e=\frac{1}{|H|} \sum_{h \in H} h
$$

is the principal block idempotent of $K G$ and of $F G$ (in particular, $s=1$ ). The nilpotent ideal $e J(F P)$ of $F A$ is contained in $J(F A)$. Hence by Villamayor's Theorem (cf. [5], 11.8),

$$
e J(F P)=e J(F G)=J\left(B_{1}\right)
$$

Therefore $e J(F P)$ is commutative.

To apply Burnside's criterion for $p$-nilpotence, choose arbitrary $s \in P$ and $u \in N_{G}(P)$. Putting $u s u^{-1}=s_{1} \in P$, we have

$$
\begin{aligned}
e(1-s)\left(1-s_{1}\right) & =e(1-s) e \cdot u(1-s) u^{-1}=[e(1-s) u \cdot e(1-s)] u^{-1} \\
& =e(1-s)^{2} .
\end{aligned}
$$

As $P$ is a right transversal of $H$ in $A$, this implies that

$$
(1-s)\left(1-s_{1}\right)=(1-s)^{2}
$$

hence

$$
s_{1}-s s_{1}=s-s^{2}
$$

If $p \neq 2$, this yields $s_{1}=s$. This is true for $p=2$ also, because otherwise $s s_{1}=s$ and hence $s_{1}=1=s$.

Therefore always $s_{1}=u s u^{-1}=s$ is valid, i.e. $P$ is contained in the centre of its normalizer. Burnside's criterion now finishes the proof of (iii). follows.

$($ iii $) \Rightarrow(i)$. With the preceding notation, 2.5 again gives $B_{1} \cong F G / H \cong F P$ and (i) 
5. Group algebras with commutative radicals. As an application of the theorem above we prove in this section the result of Wallace [10] characterizing group algebras with commutative radicals.

Proposition 5.1. Let $F$ be an arbitrary field of characteristic $p \neq 2$ and let the radical $J(F G)$ of the group algebra FG be commutative. Denoting by $G^{\prime}$ the commutator subgroup and by $P$ a Sylow p-subgroup of $G$ the following hold: $p$ does not divide $\left|G^{\prime}\right|$ and, setting

$$
f=\frac{1}{\left|G^{\prime}\right|} \sum_{x \in G^{\prime}} x
$$

we have $J(F G)=J(f \cdot F G)$; in particular

$$
\operatorname{dim}_{F} J(F G)=\operatorname{dim}_{F} J\left(F G / G^{\prime}\right)=(|P|-1) \frac{|G|}{\left|G^{\prime}\right||P|} .
$$

Proof. By $[5,12.12]$ there is no loss of generality in assuming for the proof of this assertion that $F$ is algebraically closed. For every block $B \leftrightarrow e \leftrightarrow \lambda$ of $F G$ with positive defect, we have $J(B)^{2} \neq 0$, because otherwise, by 3.1 , the Cartan matrix $C(B)$ of $B$ has just one entry, namely 2 , and this implies, by $[1,84.17]$, that $p=2$, contradicting our assumption. The commutativity of $J(F G)$ implies by the theorem that $G$ is $p$-nilpotent with $P$ abelian. So $p$ does not divide $\left|G^{\prime}\right|$. Clearly $f \cdot F G \cong F G / G^{\prime}$. From $F G=f \cdot F G \oplus(1-f) F G$ and the fact that the one-dimensional $F G$-modules and the irreducible (hence one-dimensional) modules of $F G / G^{\prime} \cong f \cdot F G$ correspond in a one-to-one manner, we conclude, by 3.3 , that all blocks $B \leftrightarrow e \leftrightarrow \lambda$ of $F G$ with $e(1-f)=e$ have defect zero. Hence $(1-f) F G$ is semisimple (see e.g. [3], Cor. 3.4). This yields $J(F G)=J(f \cdot F G)$.

Denoting by $\bar{P}=G^{\prime} P / G^{\prime}$ the Sylow $p$-subgroup of the abelian group $G / G^{\prime}$ it is easily shown that $J(F \bar{P}) \cdot F G / G^{\prime}=J\left(F G / G^{\prime}\right)$. Thus

$$
\operatorname{dim}_{F} J(F G)=(|P|-1) \frac{|G|}{\left|G^{\prime} P\right|} .
$$

and the assertion is proved.

We recall that a finite $p$-nilpotent group $G$ with a Sylow $p$-subgroup $P$ and normal $p$ complement $N$ is a Frobenius group for $P$, if $n \neq q n q^{-1}$ for every $1 \neq q \in P$ and every $1 \neq n \in$ $N$.

Quoting elementary facts written up for example in [5], we obtain as a corollary by means of the preceding results the following.

COROLlaRY 5.2 (Wallace [10]). Let $G$ be a finite group and let $F$ be a field with prime characteristic $p \neq 2$. Denoting by $P$ a Sylow p-subgroup and by $G^{\prime}$ the commutator subgroup of $G$, the following statements are equivalent:

(i) $J(F G) \leqq Z(F G)$,

(ii) $J(F G)$ is commutative,

(iii) $p$ does not divide $\left|G^{\prime}\right|$ and $G^{\prime} P$ is a Frobenius group for $P$. 
Proof. Clearly (i) implies (ii).

[5].

(ii) $\Rightarrow$ (iii) follows from 5.1 and Villamayor's Theorem (cf. [5], 11.8), applying 13.6 of (iii) $\Rightarrow$ (i) follows from 13.8 of [5].

\section{REFERENCES}

1. C. W. Curtis and I. Reiner, Representation theory of finite groups and associative algebras (New York, 1962).

2. P. Fong, On the characters of p-solvable groups, Trans. Amer. Math. Soc. 98 (1961), 263-284.

3. W. Hamernik, The linear character of an indecomposable module of a group algebra, J. London Math. Soc. (2) 7 (1973), 220-224. (1973)

4. S. Koczian, Zerlegungseigenschaften von Gruppenringen, Thesis, University of Munich,

5. G. O. Michler, Blocks and centers of group algebras (in: Lectures on rings and modules (Springer lecture notes in mathematics 246, 430-552, Berlin etc. 1972). 303-315.

6. G. O. Michler, The blocks of $p$-nilpotent groups over arbitrary fields, J. Algebra 24 (1973),

7. K. Morita, On group rings over a modular field which possess radicals expressible as principal ideals, Science reports of the Tokyo Bunrika Daigaku 4 (1951), 177-194.

8. H. Spiegel, Gruppenalgebren mit zentralem Radikal, Archiv d. Math. 23 (1972), 380-383.

9. H. Spiegel, Gruppenalgebren mit Hauptblockidealen mit zentralem Radikal (to be published).

10. D. A. R. Wallace, On the commutativity of the radical of a group algebra, Proc. Glasgow Math. Assoc. 7 (1965), 1-8.

\section{MATHEMATISCHES INSTITUT DER UNIVERSITÄT}

D 63 GIESSEN

ARNDTSTR. 2

W. Germany 